\title{
Message-passing for Inference and Optimization of Real Variables on Sparse Graphs
}

\author{
K. Y. Michael Wong ${ }^{1}$, C. H. Yeung ${ }^{1}$, and David Saad ${ }^{2}$ \\ ${ }^{1}$ Department of Physics, Hong Kong University of Science and Technology \\ Clear Water Bay, Hong Kong, China \\ ${ }^{2}$ NCRG, Aston University, Birmingham B4 7ET, UK \\ phkywong@ust.hk, phbill@ust.hk, d.saad@aston.ac.uk
}

\begin{abstract}
The inference and optimization in sparse graphs with real variables is studied using methods of statistical mechanics. Efficient distributed algorithms for the resource allocation problem are devised. Numerical simulations show excellent performance and full agreement with the theoretical results.
\end{abstract}

\section{Introduction}

Many inference and optimization problems make use of the graphical structures that describe the dependencies between random variables [1]. In contrast to models with extensive inter-dependencies among the variables, the graph-based models can be solved by passing messages between neighbouring variables on the graphs. This message-passing approach has gained recent success in areas such as error-correctig codes [2] and probabilistic inference [3].

Most studies so far have focused on graphs of discrete variables. However, many typical problems involve continuous variables. The main obstacle comes from the need to pass much more complicated messages among the nodes of the graphs, whereas in cases of discrete variables, the messages are countable sets of conditional probability estimates of discrete values. There have been attempts to simplify the messages for continuous variables, for example, to parametrize them using eigenfunction decomposition for special cases, but the general feasibility remains an open question [4].

In this paper we study inference and optimization problems on sparse graphs. Based on the analysis, we propose novel message-passing algorithms generally applicable to problems of continuous variables. The method is efficient since the messages consist of only the first and second derivatives of the message functions. The key to the successful simplification is that the messages to a target node are accompanied by information-provision messages from the target node, to first determine the state at which the derivatives should be calculated.

We first consider the general formulation on a sparse graph, and then examine the resource allocation problem, as a vehicle to study the principles and ingredients in message-passing. The problem is interesting for the following reasons. First, it is a well known problem in the area of distributed computing [5] to which 
significant effort has been dedicated within the computer science community. It is representative of a large class of problems in many other areas where a large number of nodes are required to balance their resources and redistribute tasks, such as reducing internet traffic congestion and streamlining network flows of commodities [6]. Many attempts were made in the computer science community, to find practical heuristic solutions to the distribution of computational load between computers connected by networks.

Second, the problem illustrates the advantages of the message-passing techniques in comparison with the much more computationally demanding global optimization techniques traditionally adopted in this family of problems, such as linear or quadratic programming [7]. For example, the computational complexity of quadratic programming for the load balancing task typically scales as the cube of the system size, whereas capitalizing on the network topology underlying the connectivity of the variables, message-passing scales linearly with the system size. An even more important advantage of message-passing techniques, relevant to practical implementation, is their distributive nature. Since they do not require a global optimizer, they are particularly suitable for distributive control in large or evolving networks.

Third, making use of the conservation of resources on graphs, the problem can be easily transformed to its dual which is exactly solvable using the price iteration scheme. This provides a benchmark for the message-passing method.

In Section 2, we analyze the problem using the Bethe approximation of statistical mechanics. We then present numerical results in Section 3, and derive the new message-passing algorithm on the basis of the analysis in Section 4 . The price iteration algorithm is presented in Section 5 for comparison. The study is extended to the unsatisfiable case in Section 6. We conclude the paper in Section 7. Early and partial work in this direction was presented in [8].

\section{The theoretical framework}

We consider a sparse graph with $N$ nodes, labelled $i=1, \ldots, N$. Each node $i$ is randomly connected to $c$ other nodes. The connectivity matrix is given by $\mathcal{A}_{i j}=1,0$ for connected and unconnected node pairs respectively. A link variable $y_{i j}$ is defined on each connected link from $j$ to $i$. We consider a cost function $E=\sum_{(i j)} \mathcal{A}_{i j} \phi\left(y_{i j}\right)+\sum_{i} \psi\left(\lambda_{i},\left\{y_{i j} \mid \mathcal{A}_{i j}=1\right\}\right)$, where $\lambda_{i}$ is a quenched variable defined on node $i$. In the context of probabilistic inference, $y_{i j}$ may represent the correlation between observables in nodes $j$ and $i, \phi\left(y_{i j}\right)$ may correspond to the logarithm of the prior distribution of $y_{i j}$, and $\psi\left(\lambda_{i},\left\{y_{i j} \mid \mathcal{A}_{i j}=1\right\}\right)$ the logarithm of the likelihood of the observables $\lambda_{i}$. In the context of resource allocation, $y_{i j} \equiv-y_{j i}$ may represent the current from node $j$ to $i, \phi\left(y_{i j}\right)$ the transportation cost, and $\psi\left(\lambda_{i},\left\{y_{i j} \mid \mathcal{A}_{i j}=1\right\}\right)$ the performance cost of the allocation task on node $i$, dependent on the node capacity $\lambda_{i}$.

We address a generic version of the resource allocation problem, in which $N$ is very large, the capacity $\lambda_{i}$ is randomly drawn from a distribution $\rho\left(\lambda_{i}\right)$, and the currents $y_{i j}$ satisfy the link bandwidth constraints $-W \leq y_{i j} \leq W$. For load 
balancing tasks, $\phi(y)$ is typically a convex function, which will be assumed in our study.

For sufficiently large $W$ and capacity distributions with non-negative average $\lambda$, there exist solutions which satisfy the capacity constraints $\sum_{j} \mathcal{A}_{i j} y_{i j}+\lambda_{i} \geq 0$. Hence we consider $\psi\left(\lambda_{i},\left\{y_{i j} \mid \mathcal{A}_{i j}=1\right\}\right)=\ln \left[\Theta\left(\sum_{j} \mathcal{A}_{i j} y_{i j}+\lambda_{i}\right)+\epsilon\right]$, where $\epsilon \rightarrow 0$, and the $\Theta$ function returns 1 for a non-negative argument and 0 otherwise. The problem reduces to the load balancing task of minimizing the cost $E=$ $\sum_{(i j)} \mathcal{A}_{i j} \phi\left(y_{i j}\right)$, subject to the capacity constraints. We call this the satisfiable case, which will be considered in Sections 3 to 5 for unconstrained links $(W=\infty)$ and $\langle\lambda\rangle>0$. The unsatisfiable case will be considered in Section 6 .

The analysis of the network is done by introducing the free energy $F=$ $-T \ln \mathcal{Z}_{y}$ for a temperature $T \equiv \beta^{-1}$, where $\mathcal{Z}_{y}$ is the partition function

$$
\mathcal{Z}_{y}=\prod_{(i j)} \int_{-W}^{W} d y_{i j} \prod_{i} \Theta\left(\sum_{j} \mathcal{A}_{i j} y_{i j}+\lambda_{i}\right) \exp \left[-\beta \sum_{(i j)} \mathcal{A}_{i j} \phi\left(y_{i j}\right)\right] .
$$

When the connectivity $c$ is low, the probability of finding a loop of finite length on the graph is low, and the Bethe approximation well describes the local environment of a node. In the approximation, a node is connected to $c$ branches in a tree structure, and the correlations among the branches of the tree are neglected. In each branch, nodes are arranged in generations. A node is connected to an ancestor node of the previous generation, and another $c-1$ descendent nodes of the next generation. Thus, the node is the vertex of the tree structure formed by its descendents.

Consider a vertex $V(\mathbf{T})$ of a tree $\mathbf{T}$ having a capacity $\lambda_{V(\mathbf{T})}$, and a current $y$ is drawn from the vertex by its ancestor. One can write an expression for the free energy $F(y \mid \mathbf{T})$ as a function of the free energies $F\left(y_{k} \mid \mathbf{T}_{k}\right)$ of its descendants, that branch out from this vertex, where $\mathbf{T}_{k}$ represents the tree terminated at the $k^{\text {th }}$ descendent of the vertex. The free energy can be considered as the sum of two parts, $F(y \mid \mathbf{T})=N_{\mathbf{T}} F_{\text {av }}+F_{V}(y \mid \mathbf{T})$, where $N_{\mathbf{T}}$ is the number of nodes in the tree $\mathbf{T}, F_{\text {av }}$ is the average free energy per node, and $F_{V}(y \mid \mathbf{T})$ is referred to as the vertex free energy. Note that when a vertex is added to a tree, there is a change in the free energy due to the added vertex. Since the number of nodes increases by 1 , the vertex free energy is obtained by subtracting the free energy change by the average free energy. This allows us to obtain the recursion relation

$$
\begin{aligned}
F_{V}(y \mid \mathbf{T})= & -T \ln \left\{\prod_{k=1}^{c-1}\left(\int_{-W}^{W} d y_{k}\right) \Theta\left(\sum_{k=1}^{c-1} y_{k}-y+\lambda_{V(\mathbf{T})}\right)\right. \\
& \left.\times \exp \left[-\beta \sum_{k=1}^{c-1}\left(F_{V}\left(y_{k} \mid \mathbf{T}_{k}\right)+\phi\left(y_{k}\right)\right)\right]\right\}-F_{\mathrm{av}} .
\end{aligned}
$$


For optimization, we take the zero temperature limit of Eq. (2), in which the free energy reduces to the minimum cost, yielding

$$
F_{V}(y \mid \mathbf{T})=\min _{\left\{y_{k} \mid \sum_{k=1}^{c-1} y_{k}-y+\lambda_{V(\mathbf{T})} \geq 0\right\}}\left[\sum_{k=1}^{c-1}\left(F_{V}\left(y_{k} \mid \mathbf{T}_{k}\right)+\phi\left(y_{k}\right)\right)\right]-F_{\mathrm{av}} .
$$

These iterative equations can be directly linked to those obtained from a principled Bayesian approximation, where the logarithms of the messages passed between nodes are proportional to the vertex free energies.

The current distribution and the average cost per link can be derived by integrating the current $y^{\prime}$ in a link from one vertex to another, fed by the trees $\mathbf{T}_{1}$ and $\mathbf{T}_{2}$, respectively; the obtained expressions are $P(y)=\left\langle\delta\left(y-y^{\prime}\right)\right\rangle_{\star}$ and $\langle\phi\rangle=\left\langle\phi\left(y^{\prime}\right)\right\rangle_{\star}$ where

$$
\langle\bullet\rangle_{\star}=\left\langle\frac{\int d y^{\prime} \exp \left[-\beta\left(F_{V}\left(y^{\prime} \mid \mathbf{T}_{1}\right)+F_{V}\left(-y^{\prime} \mid \mathbf{T}_{2}\right)+\phi\left(y^{\prime}\right)\right)\right](\bullet)}{\int d y^{\prime} \exp \left[-\beta\left(F_{V}\left(y^{\prime} \mid \mathbf{T}_{1}\right)+F_{V}\left(-y^{\prime} \mid \mathbf{T}_{2}\right)+\phi\left(y^{\prime}\right)\right)\right]}\right\rangle_{\lambda} .
$$

Before closing this section, we mention the alternative analysis of the problem using the replica method [9], which was successfully applied in the physics of disordered systems. The derivation is rather involved (details will be provided elsewhere), but gives rise to the same recursive equation Eq. (2) as in the Bethe approximation.

\section{Numerical solution}

The Bethe approximation provides a theoretical tool to analyze the properties of optimized networks. The solution of Eq. (3) is free from finite size effects inherent in Monte Carlo simulations, and can be obtained numerically. Since the vertex free energy of a node depends on its own capacity and the disordered configuration of its descendants, we generate 1000 nodes at each iteration of Eq. (3), with capacities randomly drawn from the distribution $\rho(\lambda)$, each being fed by $c-1$ nodes randomly drawn from the previous iteration. We have discretized the vertex free energies $F_{V}(y \mid \mathbf{T})$ function into a vector, whose $i^{\text {th }}$ component takes the value $F_{V}\left(y_{i} \mid \mathbf{T}\right)$.

To compute the average cost, we randomly draw 2 nodes, compute the optimal current flowing between them, and repeat the process 1000 times to obtain the average. Figure 1(a) shows the results as a function of iteration step $t$, for a Gaussian capacity distribution $\rho(\lambda)$ with variance 1 and average $\langle\lambda\rangle$. Each iteration corresponds to adding one extra generation to the tree structure, such that the iterative process corresponds to approximating the network by an increasingly extensive tree. We observe that after an initial rise with iteration steps, the average energies converge to steady-state values, at a rate which increases with the average capacity.

To study the convergence rate of the iterations, we fit the average cost at iteration step $t$ using $\langle E(t)-E(\infty)\rangle \sim \exp (-\gamma t)$ in the asymptotic regime. As 
shown in the inset of Fig. 1(a), the relaxation rate $\gamma$ increases with the average capacity. It is interesting to note that a cusp exists at the average capacity of about 0.45 . Below that value, convergence of the iteration is slow, since the average cost curve starts to develop a plateau before the final convergence. On the other hand, the plateau disappears and the convergence is fast above the cusp. The slowdown of convergence below the cusp is probably due to the appearance of increasingly large clusters of saturated nodes on the network, since clusters of nodes with negative capacities become increasingly extensive, and need to draw currents from increasingly extensive regions of nodes with excess capacities to satisfy the demand.
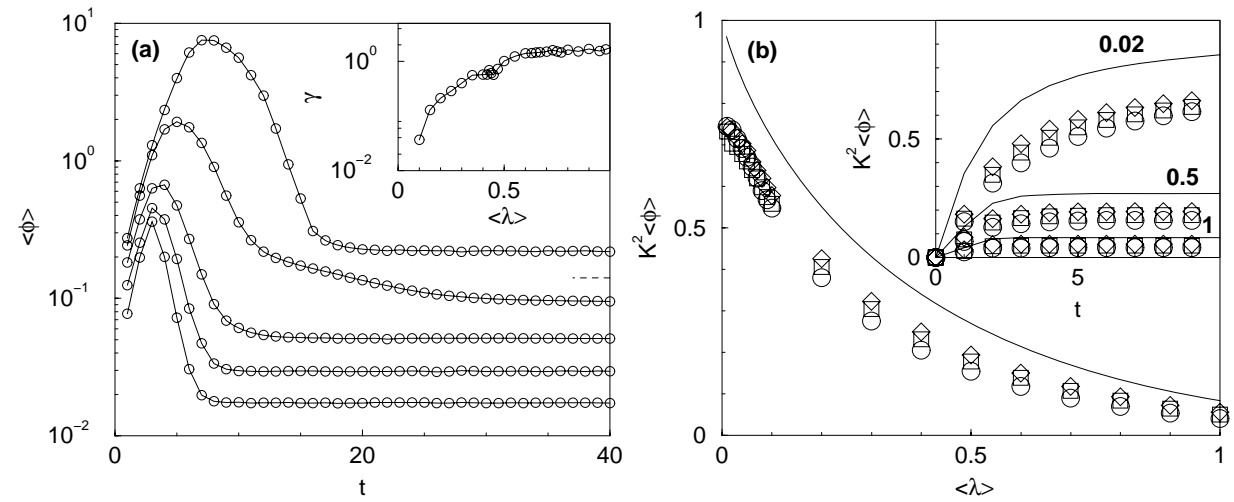

Fig. 1. Results for $N=1000, \phi(y)=y^{2} / 2$ and $W=\infty$. (a) $\langle\phi\rangle$ obtained by iterating Eq. (2) as a function of $t$ for $\langle\lambda\rangle=0.1,0.2,0.4,0.6,0.8$ (top to bottom), $c=3$ and 200800 samples. Dashed line: the asymptotic $\langle\phi\rangle$ for $\langle\lambda\rangle=0.1$. Inset: $\gamma$ as a function of $\langle\lambda\rangle$. (b) $K^{2}\langle\phi\rangle$ as a function of $\langle\lambda\rangle$ for $c=3(\bigcirc), 4(\square), 5(\diamond)$ and 1000 samples. Line: large $K$. Inset: $K^{2}\langle\phi\rangle$ as a function of time for random sequential update of Eqs. (5-6). Symbols: as in (b) for $\langle\lambda\rangle=0.02,0.1,0.5$ (top to bottom).

\section{The message-passing algorithm}

The local nature of the recursion relation Eq. (3) points to the possibility that the network optimization can be solved by local iterative approaches. However, in contrast to other message-passing algorithms which pass conditional probability estimates of discrete variables to neighboring nodes, the messages in the present context are more complex, since they are functions $F_{V}(y \mid \mathbf{T})$ of the current $y$. We simplify the message to 2 parameters, namely, the first and second derivatives of the vertex free energies. For the quadratic load balancing task, it can be shown that a self-consistent solution of the recursion relation, Eq. (3), consists of vertex free energies which are piecewise quadratic with continuous slopes. This makes the 2-parameter message a very precise approximation. 
Let $\left(A_{i j}, B_{i j}\right) \equiv\left(\partial F_{V}\left(y_{i j} \mid \mathbf{T}_{j}\right) / \partial y_{i j}, \partial^{2} F_{V}\left(y_{i j} \mid \mathbf{T}_{j}\right) / \partial y_{i j}^{2}\right)$ be the message passed from node $j$ to $i$; using Eq.(3), the recursion relation of the messages become

$$
\begin{aligned}
& A_{i j} \leftarrow-\mu_{i j}, \quad B_{i j} \leftarrow \Theta\left(-\mu_{i j}\right)\left[\sum_{k \neq i} \mathcal{A}_{j k}\left(\phi_{j k}^{\prime \prime}+B_{j k}\right)^{-1}\right]^{-1}, \\
& \mu_{i j}=\min \left[\frac{\sum_{k \neq i} \mathcal{A}_{j k}\left[y_{j k}-\left(\phi_{j k}^{\prime}+A_{j k}\right)\left(\phi_{j k}^{\prime \prime}+B_{j k}\right)^{-1}\right]-y_{i j}+\lambda_{j}}{\sum_{k \neq i} \mathcal{A}_{j k}\left(\phi_{j k}^{\prime \prime}+B_{j k}\right)^{-1}}, 0\right],
\end{aligned}
$$

with $\phi_{j k}^{\prime}$ and $\phi_{j k}^{\prime \prime}$ representing the first and second derivatives of $\phi(y)$ at $y=y_{j k}$ respectively. The forward passing of the message from node $j$ to $i$ is followed by a backward message from node $j$ to $k$ for updating the currents $y_{j k}$ according to

$$
y_{j k} \leftarrow y_{j k}-\frac{\phi_{j k}^{\prime}+A_{j k}+\mu_{i j}}{\phi_{j k}^{\prime \prime}+B_{j k}} .
$$

We note that Eqs. (5-6) differ from conventional message-passing algorithms in that backward messages of the currents are present. As a consequence of representing the messages by the first and second derivatives, the backward messages serve to inform the descendent nodes of the particular arguments they should use in calculating the derivatives for sending the next messages. Furthermore, the criterion that $y_{i j}=-y_{j i}$ provides a check for the convergence of the algorithm.

The message-passing equations further enable us to study the properties of the optimized networks in the limit of large $K \equiv c-1$, and hence consider the convergence to this limit when the connectivity increases. Given an arbitrary cost function $\phi$ with nonvanishing second derivatives for all arguments, Eq. (3) converges in the large $K$ limit to the steady-state results $A_{i j}=$ $\max \left(\left[\sum_{k \neq i} \mathcal{A}_{j k} A_{j k}-\lambda_{j}\right] / K, 0\right), B_{i j} \sim K^{-1}$. Then, $\sum_{k \neq i} \mathcal{A}_{j k} A_{j k}$ becomes selfaveraging and equal to $K m_{A}$, where $m_{A} \sim K^{-1}$ is the mean of the messages $A_{i j}$ given by $K m_{A}=\langle\Theta(x-\lambda)(x-\lambda)\rangle_{\lambda}$. Thus, $y_{i j} \sim \mu_{i} \sim K^{-1}$. The physical picture of this scaling behavior is that the current drawn by a node is shared among the $K$ descendent nodes. After rescaling, quantities such as $K^{2}\langle\phi\rangle, P(K y) / K$ and $P(K \mu) / K$ become purely dependent on the capacity distribution $\rho(\lambda)$.

For increasing finite values of $K$, Fig. 1(b) shows the common trend of $K^{2}\langle\phi\rangle$ decreasing with $\langle\lambda\rangle$ exponentially, and gradually approaching the large $K$ limit. The scaling property extends to the optimization dynamics (Fig. 1(b) inset). As shown in Fig. 2(a), the current distribution $P(K y) / K$ consists of a delta function component at $y=0$ and a continuous component, whose breadth decreases with $\langle\lambda\rangle$. Remarkably, the distributions for different connectivities collapse almost perfectly after the currents are rescaled by $K^{-1}$, with a very mild dependence on $K$ and gradually approaching the large $K$ limit. As shown in the inset of Fig. 2(a), the fraction of idle links increases with $\langle\lambda\rangle$. The fraction has a weak dependence on the connectivity, confirming the almost universal distributions rescaled for different $K$.

Since the current on a link scales as $K^{-1}$, the allocated resource of a node should have a weak dependence on the connectivity. Defining the resource at 

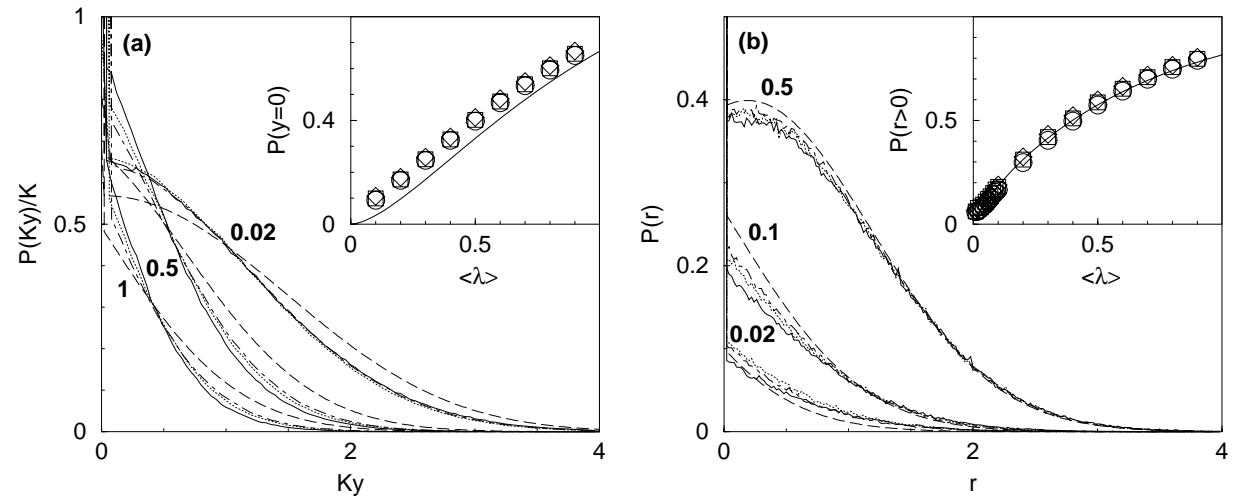

Fig. 2. Results for $N=1000, \phi(y)=y^{2} / 2, W=\infty$ and 1000 samples. (a) The current distribution $P(K y) / K$ for $\langle\Lambda\rangle=0.02,0.5,1$, and $c=3$ (solid lines), 4 (dotted lines), 5 (dot-dashed lines), large $K$ (long dashed lines). Inset: $P(y=0)$ as a function of $\langle\lambda\rangle$ for $c=3(\bigcirc), 4(\square), 5(\diamond)$, large $K$ (line). (b) The resource distribution $P(r)$ for $\langle\lambda\rangle=0.02,0.1,0.5$, large $K$. Symbols: as in (a). Inset: $P(r>0)$ as a function of $\langle\lambda\rangle$. Symbols: as in the inset of (a).

node $i$ by $r_{i} \equiv \sum_{j} \mathcal{A}_{i j} y_{i j}+\lambda_{i}$, the resource distribution $P(r)$ shown in Fig. 2(b) confirms this behavior even at low connectivities. The fraction of nodes with unsaturated capacity constraints increases with the average capacity, and is weakly dependent on the connectivity (Fig. 2(b) inset). Hence the saturated nodes form a percolating cluster at a low average capacity, and breaks into isolated clusters at a high average capacity. It is interesting to note that at the average capacity of 0.45 , below which a plateau starts to develop in the relaxation rate of the recursion relation, Eq. (3), the fraction of saturated nodes is about 0.47, close to the theoretical percolation threshold of 0.5 for $c=3$.

\section{The price iteration algorithm}

An alternative distributed algorithm can be obtained by iterating the chemical potentials of the node. Introducing Lagrange multipliers $\mu_{i}$ for the capacity constraints we get, for links with unlimited bandwidths, $L=\sum_{(i j} \mathcal{A}_{i j} \phi\left(y_{i j}\right)+$ $\sum_{i}\left(\sum_{j} \mathcal{A}_{i j} y_{i j}+\lambda_{i}\right)$. The extremum condition yields $y_{i j}=\phi^{\prime-1}\left(\mu_{j}-\mu_{i}\right)$, and using the Kühn-Tucker condition, $\mu_{i}$ can be solved in terms of $\mu_{j}$ of its neighbours, namely,

$$
\mu_{i}=\min \left(g_{i}^{-1}(0), 0\right) ; \quad g_{i}(x)=\sum_{j} \mathcal{A}_{i j} \phi^{\prime-1}\left(\mu_{j}-x\right)+\lambda_{i} .
$$

This provides a local iterative method for the optimization problem. We may interpret this algorithm as a price iteration scheme, by noting that the Lagrangian can be written as $L=\sum_{(i j)} \mathcal{A}_{i j} L_{i j}+$ constant, where $L_{i j}=\phi\left(y_{i j}\right)+\left(\mu_{i}-\mu_{j}\right) y_{i j}$. 
Therefore, the problem can be decomposed into independent optimization subproblems, each for a current on a link. $\mu_{i}$ is the storage price at node $i$, and each subproblem involves balancing the transportation cost on the link, and the storage cost at node $i$ less that at node $j$, yielding the optimal solution. This provides a pricing scheme for the individual links to optimize, which simultaneously optimize the global performance [10]. Simulations show that it yields excellent agreement with the theory Eq. (3) and message-passing Eqs. (5-6).

\section{The unsatisfiable case}

For links with small bandwidth $W$, or nodes with negative average capacity, there exist nodes which violate the capacity constraint. In these unsatisfiable cases, it is expedient to relax the constraints and search for optimal solutions which limit the violations. Hence we consider the cost $\psi\left(\lambda_{i},\left\{y_{i j} \mid \mathcal{A}_{i j}=1\right\}\right)=$ $\Theta\left(-\sum_{j} \mathcal{A}_{i j} y_{i j}-\lambda_{i}\right)\left(\sum_{j} \mathcal{A}_{i j} y_{i j}+\lambda_{i}\right)^{2} / 2$. The message-passing algorithm now becomes

$$
\begin{aligned}
& A_{i j} \leftarrow-\mu_{i j}, \\
& B_{i j} \leftarrow\left\{1+\sum_{k \neq i} \mathcal{A}_{j k}\left(\phi_{j k}^{\prime \prime}+B_{j k}\right)^{-1} \Theta\left[W-\left|y_{j k}-\frac{\phi_{j k}^{\prime}+A_{j k}+\mu_{i j}}{\phi_{j k}^{\prime \prime}+B_{j k}}\right|\right]\right\}^{-1}
\end{aligned}
$$

where $\mu_{i j}=\min \left(g_{i j}^{-1}(0), 0\right)$, with

$$
g_{i j}(x)=\sum_{k \neq i} \mathcal{A}_{j k} \max \left\{-W, \min \left[W, \phi^{\prime-1}\left(\mu_{j k}-x\right)\right]\right\}-y_{i j}+\lambda_{j}-x,
$$

The backward message is given by

$$
y_{j k} \leftarrow \max \left[-W, \min \left(W, y_{j k}-\frac{\phi_{j k}^{\prime}+A_{j k}+\mu_{i j}}{\phi_{j k}^{\prime \prime}+B_{j k}}\right)\right] .
$$

The price iteration algorithm now uses $\mu_{i}=\min \left(g_{i}^{-1}(0), 0\right)$, where

$$
g_{i}(x)=\sum_{j} \mathcal{A}_{i j} \max \left\{-W, \min \left[W, \phi^{\prime-1}\left(\mu_{j}-x\right)\right]\right\}+\lambda_{i}-x,
$$

As shown in Fig. 3(a), the average cost per node $\langle E\rangle / N$ increases rapidly when $\langle\lambda\rangle$ enters the unsatisfiable regime, and the results obtained by the theory, the message-passing and price iteration algorithms show excellent agreement. There are 3 types of links in the network: idle $\left(\left|y_{i j}\right|=0\right)$, unsaturated $\left(\left|y_{i j}\right|<W\right)$ and saturated $\left(\left|y_{i j}\right|=W\right)$. When $\langle\lambda\rangle$ enters the unsatisfiable regime, the fraction of idle links vanishes rapidly, while that of saturated links increases to a steady level, implying that more resources are transported in the links in response to the networkwide demand on resources (Fig. 3(a) inset). 
Figure $3(\mathrm{~b})$ shows the simulation results when $W$ varies. For large values of $W$, the average cost is effectively constant, since the link bandwidth constraints become irrelevant. On the other hand, when $W$ decreases, the average cost increases rapidly, since the links become increasingly ineffective in allocating resources in the network.

As shown in Fig. 3(b) inset, the fraction of saturated links increases when $W$ decreases. It is interesting to note that the fraction of idle links increases when $W$ decreases, contrary to the expectation that more links are involved in resource provision. This can be attributed to what we call a relay effect. If the links in the network were unconstrained, nodes with sufficiently large violations would have drawn currents from distant neighbours, causing currents to flow through many intermediate nodes, which act as relays for resource transmission. However, when $W$ is small, the currents drawn by nodes with violations from their nearest neighbours may have already saturated the links, and there is no use to draw currents from further neighbours. In the limit of vanishing $W$, the links are exclusively either idle or saturated. In this limit, a link is idle only when both nodes at its ends have positive $\lambda$. Hence the fraction of idle links is $f_{\text {idle }}=1-f_{\text {sat }}=[P(\lambda>0)]^{2}$. Since the transportation cost is negligible in this limit, the contribution to the average cost only comes from the violated nodes, given by $\langle E\rangle / N=\left\langle\Theta(-\lambda) \lambda^{2} / 2\right\rangle_{\lambda}$. These predictions are consistent with the simulation results in Fig. 3(b).
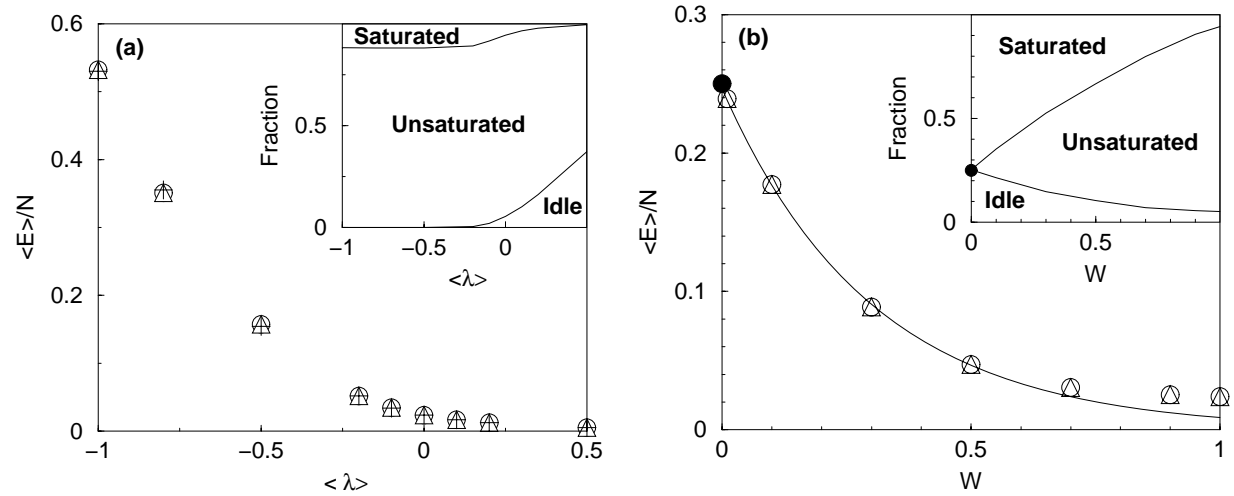

Fig. 3. Results for $N=1000, c=3, \phi=0.05 y^{2}$ and 100 samples. (a) $\langle E\rangle / N$ as a function of $\langle\lambda\rangle$ for $W=1$. Symbols: Bethe approximation $(+)$, message-passing $(\triangle)$, price iteration $(\bigcirc)$. Inset: the fraction of idle, unsaturated and saturated links as a function of $\langle\lambda\rangle$ for $W=1$; the vertical height of each region for a given $\langle\lambda\rangle$ corresponds to the respective fraction. (b) $\langle E\rangle / N$ as a function of $W$ for $\langle\lambda\rangle=0$. Symbols: messagepassing $(\triangle)$, price iteration $(\bigcirc), W \rightarrow 0$ theoretical limit $(\bullet)$. Line: exponential fit for small values of $W$. Inset: the fraction of idle, unsaturated and saturated links as a function of $W$ for $\langle\lambda\rangle$. Symbol: $W \rightarrow 0$ theoretical limit of the fraction of idle links $(\bullet)$. 


\section{Conclusion}

We have studied a prototype problem of resource allocation on sparsely connected graphs. The resultant recursion relation leads to a message-passing algorithm for optimizing the average cost, which significantly reduces the computational complexity of the existing method of global optimization, and is suitable for online distributive control. The suggested 2-parameter approximation produces results with excellent agreement with the original recursion relation and the price iteration algorithm. The Bethe approximation also reveals the scaling properties of this model, showing that the resource distribution on the nodes depends principally on the networkwide availability of resources, and depends only weakly on the connectivity. Links share the task of resource provision, leading to current distributions that are almost universally dependent on the resource availability after rescaling.

While the analysis focused on fixed connectivity and zero temperature for optimization, it can accommodate any connectivity profile and temperature parameter. For instance, we have considered the effects of adding anharmonic terms and frictional terms to the quadratic cost function. The message-passing algorithm can be adapted to these variations, and the results will be presented elsewhere. Besides, it can be used for analyzing a range of inference problems with continuous variables other than optimization. These advances open up a rich area for further investigations with many potential applications in optimization and inference.

Acknowledgments This work is partially supported by research grants HKUST 6062/02P, DAG04/05.SC25 and DAG05/06.SC36 of the Research Grant Council of Hong Kong and by EVERGROW, IP No. 1935 in the FET, EU FP6 and STIPCO EU FP5 contract HPRN-CT-2002-00319.

\section{References}

1. Jordan M. I. (ed.), Learning in Graphical Models, MIT Press, Cambridge, MA (1999).

2. Opper M. and Saad D., Advanced Mean Field Methods, MIT press (2001).

3. MacKay D.J.C., Information Theory, Inference and Learning Algorithms, CUP UK (2003).

4. Skantzos N. S., Castillo I. P. and Hatchett J. P. L., arXiv:cond-mat/0508609 (2005).

5. Peterson L. and Davie B.S., Computer Networks: A Systems Approach, Academic Press, San Diego, CA (2000).

6. Shenker S., Clark D., Estrin D. and Herzog S., ACM Computer Comm. Review 26 (1996) 19.

7. Bertsekas D., Linear Network Optimzation, MIT Press, Cambridge, MA (1991).

8. Wong K. Y. M. and Saad D., arXiv:cond-mat/0509794 (2005).

9. Mézard M., Parisi P. and Virasoro M., Spin Glass Theory and Beyond, World Scientific, Singapore (1987).

10. Kelly F. P., Euro. Trans. on Telecommunications $\&$ Related Technologies 8 (1997) 33. 